\title{
Weak solutions to boundary value problems for fractional differential equations via variational methods
}

\author{
Peiluan $\mathrm{Li}^{\mathrm{a}, *}$, Changjin $\mathrm{Xu}^{\mathrm{b}}$, Hui Wang ${ }^{\mathrm{c}}$ \\ ${ }^{a}$ School of Mathematics and Statistics, Henan University of Science and Technology, Luoyang, 471023, P. R. China. \\ ${ }^{b}$ Guizhou Key Laboratory of Economics System Simulation, Guizhou College of Finance and Economics, Guiyang, 550004, P. R. \\ China. \\ ${ }^{c}$ College of Information Engineering, Henan University of Science and Technology, Luoyang, 471003, P. R. China.
}

Communicated by R. Saadati

\begin{abstract}
Using variational methods, we investigate the solutions to the boundary value problems for fractional order differential equations. First, we consider the eigenvalue problem associated with it. Then, we obtain the existence of at least two weak solutions for every real number via Brezis and Nirenberg's Linking Theorem. Furthermore, for every positive integer $k$, the existence criteria of $k$ pairs of weak solutions are established by using Clark Theorem. At last, some examples are also given to illustrate the results. (C)2016 All rights reserved.
\end{abstract}

Keywords: Fractional differential equations, critical points, variational method, eigenvalue problem. 2010 MSC: 34B37, 34K10, 26A33.

\section{Introduction}

Fractional calculus provide a powerful tool for the description of hereditary properties of various materials and memory processes [6, 24]. Fractional differential equations have recently proved to be strong tools in the modeling of medical, physics, economics and technical sciences. For more details on fractional calculus theory, one can see the monographs of Kilbas et al. [11, Lakshmikantham et al. [12], Podlubny [16] and

\footnotetext{
${ }^{*}$ Corresponding author

Email addresses: lpllpl_lpl@163.com (Peiluan Li), xcj403@126.com (Changjin Xu), wh@haust.edu.cn (Hui Wang)
} 
Tarasov [20]. Fractional differential equations are involving the Riemann-Liouville fractional derivative or the Caputo fractional derivative have been paid more and more attentions.

In recent years, some fixed point theorems and monotone iterative methods have been applied successfully to investigate the existence of solutions for nonlinear fractional boundary value problems, see for example, [1, 2, 7, 14, 22, and the references therein. On the other hand, critical point theory and the variational methods have been very useful in dealing with the existence and multiplicity of solutions for integer order differential equations with some boundary conditions. But until now, there are few works that deal with the fractional differential equations via the variational methods; see [3, 5, 8, 10, 13, 15, 18, 19, 21, 23]. It is often very difficult to establish a suitable space and variational functional for fractional boundary value problem for several reasons. First and foremost, the composition rule in general fails to be satisfied by fractional integral and fractional derivative operators. Furthermore, the fractional integral is a singular integral operator and fractional derivative operator is non-local. Besides, the adjoint of a fractional differential operator is not the negative of itself. By means of critical point theory, Jiao and Zhou [9] considered the following fractional boundary value problems

$$
\left\{\begin{array}{l}
{ }_{t} D_{T}^{\alpha}\left({ }_{0} D_{t}^{\alpha} u(x)\right)=\nabla F(t, u(t)), \text { a.e. } t \in[0, T], \\
u(0)=u(T)=0,
\end{array}\right.
$$

where $\alpha \in(0,1),{ }_{0} D_{t}^{\alpha}$ and ${ }_{t} D_{T}^{\alpha}$ are the left and right Riemann-Loiuville fractional derivatives respectively. $F:[0, T] \times R^{N} \rightarrow R$ ( with $N \geq 1$ ) is a suitable given function and $\nabla F(t, x)$ is the gradient of $F$ with respect to $x$.

In [13, the authors investigated the following nonlinear fractional boundary value problems

$$
\begin{aligned}
-\frac{d}{d t}\left(\frac{1}{2}{ }_{0} D_{t}^{\beta}+\frac{1}{2}{ }_{t} D_{T}^{\beta}\right) u^{\prime}(t) & =\lambda u(t)+\nabla F(t, u(t))=0, \\
u(0) & =u(T)=0,
\end{aligned}
$$

where $\beta \in(0,1),{ }_{0} D_{t}^{\beta}$ and ${ }_{t} D_{T}^{\beta}$ are the left and right Rieman-Liouville fractional integrals of order $\beta$ respectively, and $\lambda \in R$ is a parameter, $F:[0, T] \times R^{N} \rightarrow R$ is a suitable given function and $\nabla F(t, x)$ is the gradient of $F$ with respect to $x$. The main tools are variational methods and critical point theorems.

In [3], the authors studied the following nonlinear fractional boundary value problems

$$
\left\{\begin{array}{l}
{ }_{t} D_{T}^{\alpha}\left({ }_{0} D_{t}^{\alpha} u(x)\right)=\lambda a(t) f(u(t))+\mu g(t, u(t)), \quad \text { a.e. } t \in[0, T], \\
u(0)=u(T)=0,
\end{array}\right.
$$

where $\alpha \in(0,1],{ }_{0} D_{t}^{\beta}$ and ${ }_{t} D_{T}^{\beta}$ are the left and right Rieman-Liouville fractional integrals of order $\alpha$ respectively, and $\lambda$ and $\mu$ are non-negative parameters, $a:[0, T] \rightarrow R, f: R \rightarrow R, g:[0, T] \rightarrow R$ are three given continuous functions. By means of the variational methods and a critical point theorem, the authors get the existence of infinitely many solutions.

Motivated by the work above, in this article, we consider the following fractional differential equations

$$
\left\{\begin{array}{l}
{ }_{t} D_{T}^{\alpha}\left(a(t){ }_{0} D_{t}^{\alpha} u(x)\right)=\lambda u(t) \nabla F(t, u(t)), \text { a.e. } t \in[0, T], \\
u(0)=u(T)=0,
\end{array}\right.
$$

where $\alpha \in(0,1],{ }_{0} D_{t}^{\beta}$ and ${ }_{t} D_{T}^{\beta}$ are the left and right Rieman-Liouville fractional integrals of order $\alpha$ respectively, and $\lambda \in R$ is a parameter, $F:[0, T] \times R^{N} \rightarrow R$, and $\nabla F(t, x)$ is the gradient of $F$ with respect to $x, a:[0, T] \rightarrow R$ is a continuous function.

First, we consider the eigenvalue problem associated with it. A characterization of the first eigenvalue is also given. Then, we obtain the existence of at least two weak solutions for every real number via Brezis and Nirenberg's Linking Theorem. Furthermore, for every positive integer $k$, the existence criteria of $k$ pairs 
of weak solutions are established by using Clark Theorem. At last, some examples are given to illustrate the results. Our methods and results are different from those in [3]. The rest of this paper is organized as follows. In Section 2, some definitions and lemmas which are essential to prove our main results are stated. In Section 3, we give the main results. And, two examples are offered to demonstrate the application of our main results.

\section{Preliminaries}

This section is devoted to introduce some necessary definitions and properties of the fractional calculus, which are used further in this paper.

Definition 2.1 ([1] $)$. Let $f$ be a function defined on $[a, b]$. The left and right Riemann-Liouville fractional derivatives of order $\alpha$ for function $f$ denoted by ${ }_{a} D_{t}^{\alpha}$ and ${ }_{t} D_{b}^{\alpha}$ function, respectively, are defined by

$$
\begin{aligned}
& { }_{a} D_{t}^{\alpha} f(t)=\frac{d^{n}}{d t^{n}}{ }_{a} D_{t}^{\alpha-n} f(t)=\frac{1}{\Gamma(n-\alpha)} \frac{d^{n}}{d t^{n}} \int_{a}^{t}(t-s)^{n-\alpha-1} f(s) d s, \quad t \in[a, b], \alpha>0, \\
& { }_{t} D_{b}^{\alpha} f(t)=(-1)^{n} \frac{d^{n}}{d t^{n}}{ }_{t} D_{b}^{\alpha-n} f(t)=\frac{(-1)^{n}}{\Gamma(n-\alpha)} \frac{d^{n}}{d t^{n}} \int_{t}^{b}(s-t)^{n-\alpha-1} f(s) d s, \quad t \in[a, b], \alpha>0,
\end{aligned}
$$

provided that the right-hand side integral is pointwise defined on $[a, b]$.

Definition 2.2 ([11]). If $\alpha \in(n-1, n)$ and $f \in A C^{n}([a, b], R)$, then the left and right Caputo fractional derivatives of order $\alpha$ of a function $f$ denoted by ${ }_{a} D_{t}^{\alpha} f(t)$ and ${ }_{t} D_{b}^{\alpha} f(t)$ function, respectively, are defined by

$$
\begin{aligned}
& { }_{a}^{C} D_{t}^{\alpha} f(t)={ }_{a} D_{t}^{\alpha-n} \frac{d^{n}}{d t^{n}} f(t)=\frac{1}{\Gamma(n-\alpha)} \int_{a}^{t}(t-s)^{n-\alpha-1} f^{(n)}(s) d s, \quad t \in[a, b], \alpha>0, \\
& { }_{t}^{C} D_{b}^{\alpha} f(t)=(-1)^{n}{ }_{t} D_{b}^{\alpha-n} \frac{d^{n}}{d t^{n}} f(t)=\frac{(-1)^{n}}{\Gamma(n-\alpha)} \int_{t}^{b}(s-t)^{n-\alpha-1} f^{(n)}(s) d s, \quad t \in[a, b], \alpha>0,
\end{aligned}
$$

where $t \in[a, b]$.

Lemma 2.3 ([11]). The left and right Riemann-Liouville fractional integral operators have the property of a semigroup; that is,

$$
\int_{a}^{b}\left[{ }_{a} D_{t}^{-\alpha} f(t)\right] g(t) d t=\int_{a}^{b}\left[{ }_{t} D_{b}^{-\alpha} g(t)\right] f(t) d t, \quad \alpha>0,
$$

provided that $f \in L^{p}([a, b], R), g \in L^{q}([a, b], R)$ and $p \geq q, q \geq 1, \frac{1}{p}+\frac{1}{q} \leq 1+\alpha$ or $p \neq 1, q \neq 1, \frac{1}{p}+\frac{1}{q}=1+\alpha$.

Lemma 2.4 ([11]). The left and right Riemann-Liouville fractional integral operators have the property of a semigroup; that is,

$$
\int_{a}^{b}\left[{ }_{a} D_{t}^{-\alpha} f(t)\right] g(t) d t=\int_{a}^{b}\left[{ }_{t} D_{b}^{-\alpha} g(t)\right] f(t) d t, \quad \alpha>0,
$$

provided that $f(a)=f(b)=0, f^{\prime} \in L^{\infty}\left([a, b], R^{N}\right)$ and $g \in L^{1}\left([a, b], R^{N}\right)$ or $g(a)=g(b)=0, f^{\prime} \in$ $L^{\infty}\left([a, b], R^{N}\right)$ and $f \in L^{1}\left([a, b], R^{N}\right)$.

Lemma 2.5 ([11]). Assume that $n-1<\alpha<n$ and $f \in C^{n}[a, b]$. Then

$$
\begin{aligned}
& { }_{a} D_{t}^{-\alpha}\left({ }_{a}^{C} D_{t}^{\alpha} f(t)\right)=f(t)-\sum_{j=0}^{n-1} \frac{f^{(j)}(a)}{j !}(t-a)^{j}, \\
& { }_{t} D_{b}^{-\alpha}\left({ }_{t}^{C} D_{b}^{\alpha} f(t)\right)=f(t)-\sum_{j=0}^{n-1} \frac{(-1)^{j} f^{(j)}(b)}{j !}(b-t)^{j}
\end{aligned}
$$

for $t \in[a, b]$. 
Lemma 2.6 ([11]). Assume that $n-1<\alpha<n$ and $f \in C^{n}[a, b]$. Then

$$
\begin{aligned}
& { }_{a}^{C} D_{t}^{\alpha} f(t)={ }_{a} D_{t}^{-\alpha} f(t)-\sum_{j=0}^{n-1} \frac{f^{(j)}(a)}{\Gamma(j-\alpha+1)}(t-a)^{j-\alpha}, \\
& { }_{t}^{C} D_{b}^{\alpha} f(t)={ }_{t} D_{b}^{-\alpha} f(t)-\sum_{j=0}^{n-1} \frac{(-1)^{j} f^{(j)}(b)}{\Gamma(j-\alpha+1)}(b-t)^{j-\alpha}
\end{aligned}
$$

for $t \in[a, b]$.

In order to establish a variational structure which enables us to reduce the existence of solutions of problem (1.1) to one of finding the critical points of corresponding functional, it is necessary to construct appropriate function spaces.

Let us recall that for any fixed $t \in[0, T]$ and $1 \leq p \leq \infty$,

$$
\|u\|_{\infty}=\max _{t \in[0, T]}|u(t)|, \quad\|u\|_{L^{p}}=\left(\int_{0}^{T}|u(s)|^{p} d s\right)^{\frac{1}{p}}
$$

where $T>0$ is given in 1.1 .

Let $0<\alpha \leq 1$, we define the fractional derivative spaces $E_{0}^{\alpha}$ by the closure of $C_{0}^{\infty}([0, T], R)$ with respect to the weighted norm

$$
\|u\|_{\alpha}=\left(\left.\left.\int_{0}^{T} a(t)\right|_{0} D_{t}^{\alpha} u(t)\right|^{2} d t+\int_{0}^{T}|u(t)|^{2} d t\right)^{\frac{1}{2}}, \quad \forall u \in E_{0}^{\alpha},
$$

where $C_{0}^{\infty}([0, T], R)=\left\{u \in C^{\infty}([0, T], R): u(0)=u(T)\right\}$. The fractional derivative spaces $E_{0}^{\alpha}$ is similar to the space $E_{0}^{\alpha, p}$ with $p=2$ which is defined in [9]. Clearly, the fractional derivative space $E_{0}^{\alpha}$ is the space of functions $u \in L^{2}[0, T]$ having an $\alpha$ order fractional derivative ${ }_{0} D_{t}^{\alpha} u(t) \in L^{2}[0, T]$ and $u(0)=u(T)$.

Lemma $2.7([9])$. Let $\frac{1}{2}<\alpha \leq 1$; for all $u \in E_{0}^{\alpha}$, one has

$$
\text { (I) }\|u\|_{L^{2}} \leq \frac{T^{\alpha}}{\Gamma(\alpha+1)}\left\|_{0} D_{t}^{\alpha} u(t)\right\|_{L^{2}}, \quad \text { (II) }\|u\|_{\infty} \leq \frac{T^{\alpha-1 / 2}}{\Gamma(\alpha) \sqrt{2 \alpha-1}}\left\|_{0} D_{t}^{\alpha} u(t)\right\|_{L^{2}} .
$$

Let $a(t) \geq 0$ and $a_{0}=\min _{t \in[0, T]} a(t)$, from Lemma 2.7 , one has

$$
\begin{aligned}
\|u\|_{L^{2}} & \leq \frac{T^{\alpha}}{\Gamma(\alpha+1) \sqrt{a_{0}}}\left(\left.\left.\int_{0}^{T} a(t)\right|_{0} D_{t}^{\alpha} u(t)\right|^{2} d t\right)^{\frac{1}{2}}, \\
\|u\|_{\infty} & \leq \frac{T^{\alpha-1 / 2}}{\Gamma(\alpha) \sqrt{a_{0}(2 \alpha-1)}}\left(\left.\left.\int_{0}^{T} a(t)\right|_{0} D_{t}^{\alpha} u(t)\right|^{2} d t\right)^{\frac{1}{2}} .
\end{aligned}
$$

By $(2.2),(2.3)$, we can also define

$$
\|u\|_{\alpha}=\left(\left.\left.\int_{0}^{T} a(t)\right|_{0} D_{t}^{\alpha} u(t)\right|^{2} d t\right)^{\frac{1}{2}}, \quad \forall u \in E_{0}^{\alpha} .
$$

Then we conclude that $\|u\|_{\alpha}$ defined in (2.1) is equivalent to the norm $\|u\|_{\alpha}$ defined in (2.4). In the sequel, we will consider $E_{0}^{\alpha}$ with the norm $\|u\|_{\alpha}$ defined in (2.4). Obviously, $E_{0}^{\alpha}$ is a reflexive and separable Hilbert space with the norm $\|u\|_{\alpha}$.

It follows from 2.2 -2.4 that

$$
\|u\|_{L^{2}} \leq \frac{T^{\alpha}}{\Gamma(\alpha+1) \sqrt{a_{0}}}\|u\|_{\alpha}, \quad\|u\|_{\infty} \leq \frac{T^{\alpha-1 / 2}}{\Gamma(\alpha) \sqrt{a_{0}(2 \alpha-1)}}\|u\|_{\alpha} .
$$

Similar to some properties in [9], we have the following results. 
Lemma 2.8. Let $\alpha \in(0,1]$. The fractional derivative space $E_{0}^{\alpha}$ is a reflexive and separable Banach space.

Lemma 2.9. Let $\alpha \in(0,1]$ and the sequence $\left\{u_{k}\right\}$ converges weakly to $u$ in $E_{0}^{\alpha}$. Then $u_{k} \rightarrow u$ in $C([0, T], R)$; that is, $\left\|u_{n}-u\right\|_{\infty} \rightarrow 0$, as $k \rightarrow \infty$.

Definition 2.10. $u \in E_{0}^{\alpha}$ is called a weak solution of problem (1.1) if

$$
\int_{0}^{T} a(t){ }_{0} D_{t}^{\alpha} u(t){ }_{0} D_{t}^{\alpha} v(t) d t-\lambda \int_{0}^{T} u(t) v(t) d t-\int_{0}^{T} \nabla F(t, u(t)) v(t) d t=0
$$

for all $v \in E_{0}^{\alpha}$.

Throughout this paper, we assume that the following assumption $\left(H_{1}\right)$ is satisfied.

$\left(H_{1}\right) . F(t, x)$ is continuous in $t \in[0, T]$ for every $x \in R$ and continuously differentiable in $x$ and $F(t, 0)=0$ for all $t \in[0, T], a:[0, T] \rightarrow R$ is a continuous non-negative function.

We consider the functional $I: X \rightarrow R$, defined by

$$
I(u)=\frac{1}{2} \int_{0}^{T}\left[\left.\left.a(t)\right|_{0} D_{t}^{\alpha} u(t)\right|^{2}-\lambda u^{2}(t)\right] d t-\int_{0}^{T} F(t, u(t)) d t .
$$

Then $I$ is continuously differentiable under the assumption $\left(H_{1}\right)$, and

$$
\left\langle I^{\prime}(u), v\right\rangle=\int_{0}^{T} a(t){ }_{0} D_{t}^{\alpha} u(t){ }_{0} D_{t}^{\alpha} v(t) d t-\lambda \int_{0}^{T} u(t) v(t) d t-\int_{0}^{T} \nabla F(t, u(t)) v(t) d t
$$

for all $v \in E_{0}^{\alpha}$. Hence the critical point of $I$ is the weak solution of problem (1.1). Next, we consider the critical point of $I$.

Finally, we need the following results in critical point theory.

Definition 2.11. Let $\mathrm{E}$ be a real Banach space, and $\varphi \in C^{1}(E, R)$. We say that $\varphi$ satisfies the (PS) condition if any $\left\{u_{m}\right\} \subset E$ for which $\varphi\left(u_{m}\right)$ is bounded and $\varphi^{\prime}\left(u_{m}\right) \rightarrow 0$ as $m \rightarrow \infty$ posses a convergent subsequence.

Next is the Brezis and Nirenbergs linking Theorem.

Lemma 2.12 (4]). Let $E$ have a direct sum decomposition $E=X \oplus Y$, where $\operatorname{dim} x \leq \infty$, and $\varphi$ be a $C^{1}$ functional on $E$ with $\varphi(0)=0$, satisfying $(P S)$ and assume that, for some $r>0$,

$$
\varphi(x) \leq 0, \quad \forall x \in X, \quad\|x\| \leq r, \quad \varphi(y) \geq 0, \quad \forall y \in Y, \quad\|y\| \leq r .
$$

Also assume that $\varphi$ is bounded below and $\inf _{E} \varphi<0$. Then $\varphi$ has at least two nonzero critical points.

Lemma 2.13 (Clark Theorem [17]). Let $E$ be a real Banach space, and $\varphi \in C^{1}(E, R)$, even, bounded from below and satisfying $(P S)$. Suppose $\varphi(0)=0$, there is a set $E^{\prime} \subset E$ such that $E^{\prime}$ is homeomorphic to $S^{j-1}$ ( $j-1$ dimension unit sphere) by an odd map, and $\sup _{E} \varphi<0$. Then $\varphi$ possesses at least $j$ distinct pairs of critical points.

\section{Main results}

First, we investigate the eigenvalue problem

$$
\left\{\begin{array}{l}
{ }_{t} D_{T}^{\alpha}\left(a(t){ }_{0} D_{t}^{\alpha} u(t)\right)=\lambda u(t), \quad \text { a.e. } t \in[0, T], \\
u(0)=u(T)=0
\end{array}\right.
$$

and $u \in E_{0}^{\alpha}$ is a weak solution of problem (3.1) if

$$
\int_{0}^{T} a(t)_{0} D_{t}^{\alpha} u(t){ }_{0} D_{t}^{\alpha} v(t) d t-\lambda \int_{0}^{T} u(t) v(t) d t=0
$$

for all $v \in E_{0}^{\alpha}$. 
Theorem 3.1. The eigenvalues of (3.1) are all real and can be denoted by $0<\lambda_{1} \leq \lambda_{2} \leq \cdots \leq \lambda_{k} \leq \cdots$, with $\lambda_{k} \rightarrow \infty$ as $k \rightarrow \infty$, if we repeat each eigenvalue according to its multiplicity. $\lambda_{1}$ can be characterized as

$$
\lambda_{1}=\inf _{u \in E^{\alpha} \backslash\{0\}} \frac{\left.\left.\int_{0}^{T} a(t)\right|_{0} D_{t}^{\alpha} u(t)\right|^{2} d t}{\int_{0}^{T} u^{2}(t) d t} .
$$

Furthermore, there exists an orthogonal basis $\left\{w_{k}\right\}_{k=1}^{\infty}$ of $E_{0}^{\alpha}$, where $w_{k} \in E_{0}^{\alpha}$ is a eigenfunction corresponding to $\lambda_{k}$ for $k=1,2, \ldots$.

Proof. From $\left(H_{1}\right)$, we know $a(t) \geq 0$. Then, it is easy to see $E_{0}^{\alpha}$ is a Banach space with the norm $\|u\|_{\alpha}=$ $\left(\left.\left.\int_{0}^{T} a(t)\right|_{0} D_{t}^{\alpha} u(t)\right|^{2} d t\right)^{\frac{1}{2}}, \quad \forall u \in E_{0}^{\alpha}$ which is defined in 2.4 , and an inner product can be induced by the norm $\|u\|_{\alpha}$, we denote

$$
\langle u, v\rangle_{\alpha}=\int_{0}^{T} a(t)_{0} D_{t}^{\alpha} u(t)_{0} D_{t}^{\alpha} v(t) d t .
$$

Then $E_{0}^{\alpha}$ is a Hilbert space with this inner product.

From Hölder inequality and (2.5), for given $u \in L^{2}(0, T)$ and any $v \in E_{0}^{\alpha}$, we have

$$
\left|\int_{0}^{T}\langle u, v\rangle d t\right| \leq\|u\|_{L^{2}}\|v\|_{L^{2}} \leq \frac{T^{\alpha}}{T(\alpha+1) \sqrt{a_{0}}}\|u\|_{L^{2}}\|v\|_{\alpha}
$$

According to the Riesz Theorem, there exists a unique $w \in E_{0}^{\alpha}$ such that

$$
\int_{0}^{T}\langle u, v\rangle d t=\langle w, v\rangle_{\alpha}, \quad \forall v \in E_{0}^{\alpha} .
$$

Then we can define the operator $K: L^{2}(0, T) \rightarrow E^{\alpha}$ as $K u=w$.

By the above proof, one has

$$
\|K u\|_{\alpha}=\|w\|_{\alpha} \leq \frac{T^{\alpha}}{T(\alpha+1) \sqrt{a_{0}}}\|u\|_{L^{2}} .
$$

Then it is easy to see that $K$ is a bounded linear operator from $L^{2}(0, T)$ to $E^{\alpha}$. Let $S: E^{\alpha} \rightarrow L^{2}(0, T)$ be an embedding operator. From Lemma 2.9, $S$ is compact, which shows $w=K S u$. So (3.2) can be transform into the following operator equation

$$
\langle u, v\rangle_{\alpha}=(\lambda w, v)_{\alpha}=(\lambda K S u, v)_{\alpha}, \quad \forall v \in E_{0}^{\alpha},
$$

which implies $\langle u-\lambda K S u, v\rangle_{\alpha}=0, \forall v \in E_{0}^{\alpha}$. It follows that $(I-\lambda K S) u=0$. Therefore, we can conclude $K S$ is symmetric and compact. From Lemma 2.8 , we know $E_{0}^{\alpha}$ is separable. Then by Riesz-Schauder theory, we can obtain that all eigenvalue $\left\{\lambda_{k}\right\}$ of $K S$ are positive real numbers and there are corresponding to the eigenfunctions $\left\{w_{k}\right\}_{k=1}^{\infty}$ which make up an orthogonal basis of $E^{\alpha}$, and $\lambda_{1}$ can be characterized as

$$
\lambda_{1}=\inf _{u \in E^{\alpha} \backslash\{0\}} \frac{\int_{0}^{T} a(t)\left|{ }_{0} D_{t}^{\alpha} u(t)\right|^{2} d t}{\int_{0}^{T} u^{2}(t) d t} .
$$

This completes the proof.

The following result is based on Brezis and Nirenberg's Linking Theorem.

Theorem 3.2. Let $\left(H_{1}\right)$ hold, and the following assumptions $\left(H_{2}\right)$ and $\left(H_{3}\right)$ are satisfied. $\left(H_{2}\right)$. There are constants $c_{1}>0$ and $0<p<2$ such that

$$
F(t, x) \leq-\frac{\lambda}{2}|x|^{2}+c_{1}|x|^{p}, \quad x \in R, t \in[0, T] .
$$


$\left(H_{3}\right)$. There are $k \in N$ and $r_{1}>0$ such that,

$$
\frac{\lambda_{k}-\lambda}{2}|x|^{2} \leq F(t, x), \quad \text { for }|x| \leq r_{1}, \quad t \in[0, T] .
$$

Then (1.1) possesses at least two weak solutions for $\lambda \in R$.

Proof. We show all the conditions of Lemma 2.12 are satisfied.

From $\left(H_{2}\right)$ and $(2.5)$, for $u \in E^{\alpha}$, one has

$$
\begin{aligned}
I(u) & =\frac{1}{2} \int_{0}^{T}\left[\left.\left.a(t)\right|_{0} D_{t}^{\alpha} u(t)\right|^{2}-\lambda u^{2}(t)\right] d t-\int_{0}^{T} F(t, u(t)) d t \\
& \geq \frac{1}{2}\|u\|_{\alpha}^{2}-\frac{\lambda}{2}\|u\|_{L^{2}}^{2}+\frac{\lambda}{2}\|u\|_{L^{2}}^{2}-c_{1} T\|u\|_{\infty}^{p} \\
& \geq \frac{1}{2}\|u\|_{\alpha}^{2}-c_{1} T\left(\frac{T^{\alpha-1 / 2}}{\Gamma(\alpha) \sqrt{a_{0}(2 \alpha-1)}}\|u\|_{\alpha}\right)^{p} \\
& =\left[\frac{1}{2}-c_{1}\|u\|_{\alpha}^{p-2} T\left(\frac{T^{\alpha-1 / 2}}{\Gamma(\alpha) \sqrt{a_{0}(2 \alpha-1)}}\right)^{p}\right]\|u\|_{\alpha}^{2} .
\end{aligned}
$$

From (3.4), we know $I$ is bounded from below. If $\left\{u_{n}\right\}$ is a (PS) sequence, i.e., $I\left(u_{n}\right)$ is bounded and $I^{\prime}\left(u_{n}\right) \rightarrow 0$, as $n \rightarrow \infty$, then (3.4) implies $\left\{u_{n}\right\}$ is bounded. From the reflexivity of $E^{\alpha}$, we know $\left\{u_{n}\right\}$ has a weakly convergent subsequence. Without loss of generality, we assume that $u_{k}$ converges weakly to $u$ in $E_{0}^{\alpha}$. By Lemma 2.9 , we can obtain that $u_{n} \rightarrow u$ in $C([0, T], R)$, as $n \rightarrow \infty$, that is,

$$
\left\|u_{n}-u\right\|_{\infty} \rightarrow 0, \text { as } n \rightarrow \infty \text {. }
$$

From (2.7), we have

$$
\begin{aligned}
\left\langle I^{\prime}(u), u\right\rangle & =\int_{0}^{T} a(t){ }_{0} D_{t}^{\alpha} u(t){ }_{0} D_{t}^{\alpha} u(t) d t-\lambda \int_{0}^{T} u(t) u(t) d t-\int_{0}^{T} \nabla F(t, u(t)) u(t) d t \\
& =\|u\|_{\alpha}^{2}-\lambda \int_{0}^{T} u^{2}(t) d t-\int_{0}^{T} \nabla F(t, u(t)) u(t) d t .
\end{aligned}
$$

Then

$$
\begin{aligned}
\left\|u_{n}-u\right\|_{\alpha}^{2} \leq & \left\langle I^{\prime}(u)-I^{\prime}\left(u_{n}\right), u-u_{n}\right\rangle+\lambda\left\|u_{n}-u\right\|_{\infty} \int_{0}^{T}\left|u_{n}(t)+u(t)\right| d t \\
& +\int_{0}^{T}\left(\nabla F\left(t, u_{n}(t)\right)-\nabla F(t, u(t))\right) d t\left\|u_{n}-u\right\|_{\infty} .
\end{aligned}
$$

By $\left(H_{1}\right), 3.5$, it easy to see that

$$
\int_{0}^{T}\left(\nabla F\left(t, u_{n}(t)\right)-\nabla F(t, u(t))\right) d t\left\|u_{n}-u\right\|_{\infty} \rightarrow 0, \text { as } n \rightarrow \infty .
$$

From 3.5 and $I^{\prime}\left(u_{n}\right) \rightarrow 0$ as $n \rightarrow \infty$, we have

$$
\begin{aligned}
\left\langle I^{\prime}\left(u_{n}\right)-I^{\prime}(u), u_{n}-u\right\rangle & =\left\langle I^{\prime}\left(u_{n}\right), u_{n}-u\right\rangle-\left\langle I^{\prime}(u), u_{n}-u\right\rangle \\
& \leq\left\|I^{\prime}\left(u_{n}\right)\right\|_{\alpha}\left\|u_{n}-u\right\|_{\alpha}-\left\langle I^{\prime}(u), u_{n}-u\right\rangle \rightarrow 0, \text { as } k \rightarrow \infty .
\end{aligned}
$$


By (3.5), we can also easily get

$$
\lambda\left\|u_{n}-u\right\|_{\infty} \int_{0}^{T}\left|u_{n}(t)+u(t)\right| d t \rightarrow 0, \text { as } n \rightarrow \infty .
$$

In view of (3.6)-(3.9), we know that $\left\|u_{n}-u\right\|_{\alpha} \rightarrow 0$, as $k \rightarrow \infty$. Then $u_{k}$ converges strongly to $u$ in $E_{0}^{\alpha}$. Therefore $I$ satisfies (P.S) condition.

Set $V=\operatorname{span}\left\{w_{1}, \ldots, w_{k}\right\}$ and $X=V^{\perp}$, where $\left\{w_{i}\right\}(i=1, \ldots, k)$ are eigenfunctions of (3.1). From Theorem 3.1, we know $E_{0}^{\alpha}=V \oplus X$.

For $u \in V$ with $\|u\|_{\alpha} \leq \frac{T(\alpha) \sqrt{a_{0}(2 \alpha-1)}}{T^{\alpha-1 / 2}} r_{1}$, from 2.5 , we can conclude $\|u\|_{\infty} \leq r_{1}$. Together with $\left(H_{3}\right)$, one has

$$
\begin{aligned}
I(u) & =\frac{1}{2} \int_{0}^{T}\left[\left.\left.a(t)\right|_{0} D_{t}^{\alpha} u(t)\right|^{2}-\lambda u^{2}(t)\right] d t-\int_{0}^{T} F(t, u(t)) d t \\
& \leq \frac{1}{2}\|u\|_{\alpha}^{2}-\frac{\lambda}{2} \int_{0}^{T} u^{2}(t) d t-\int_{0}^{T} \frac{\left(\lambda_{k}-\lambda\right)}{2} u^{2}(t) d t \\
& \leq \frac{1}{2}\|u\|_{\alpha}^{2}-\int_{0}^{T} \frac{\lambda_{k}}{2} u^{2}(t) d t .
\end{aligned}
$$

Theorem 3.1 implies $\lambda_{k}>\lambda_{1}$. From (3.3), we have

$$
\lambda_{k} \geq \frac{\int_{0}^{T} a(t)\left|{ }_{0} D_{t}^{\alpha} u(t)\right|^{2} d t}{\int_{0}^{T} u^{2}(t) d t}=\frac{\|u\|_{\alpha}^{2}}{\|u\|_{L^{2}}^{2}}, \text { for } u \in V,\|u\|_{\infty} \leq r_{1} .
$$

It follows from 3.10 that

$$
I(u) \leq 0, \text { for } u \in V,\|u\|_{\infty} \leq r_{1} .
$$

Notice that $X=V^{\perp}$. For $u \in X,\|u\|_{\infty} \leq r_{1}$, by $\left(H_{2}\right)$, one has

$$
\begin{aligned}
I(u) & =\frac{1}{2} \int_{0}^{T}\left[\left.\left.a(t)\right|_{0} D_{t}^{\alpha} u(t)\right|^{2}-\lambda u^{2}(t)\right] d t-\int_{0}^{T} F(t, u(t)) d t \\
& \geq \frac{1}{2}\|u\|_{\alpha}^{2}-\frac{\lambda}{2}\|u\|_{L^{2}}^{2}+\frac{\lambda}{2}\|u\|_{L^{2}}^{2}-c_{1} T\|u\|_{\infty}^{2}\|u\|_{\infty}^{p-2} \\
& \geq \frac{1}{2}\|u\|_{\alpha}^{2}\left[1-c_{1} \operatorname{Tr}_{1}^{p-2} \frac{T^{2 \alpha-1}}{\Gamma^{2}(\alpha) a_{0}(2 \alpha-1)}\right] .
\end{aligned}
$$

If we choose $r_{1} \geq\left\{c_{1} T \frac{T^{2 \alpha-1}}{\Gamma^{2}(\alpha) a_{0}(2 \alpha-1)}\right\}^{\frac{1}{2-p}}$, it follows that

$$
I(u) \geq 0, \text { for } u \in X,\|u\|_{\infty} \leq r_{1} .
$$

If $\inf _{E} I(u)<0$, all the conditions of Lemma 2.12 hold. By virtue of Lemma 2.12, we can conclude that problem (1.1) has at least two non-trivial weak solutions.

On the other hand, if $\inf _{E} I(u) \geq 0$, from 3.11, we have $I(u)=0$, for all $u \in V,\|u\|_{\infty} \leq r_{1}$. Then all $u \in V,\|u\|_{\infty} \leq r_{1}$ are weak solutions of problem (1.1).

The next results are based on Lemma 2.13 (Clark Theorem[17]).

Theorem 3.3. Suppose $\left(H_{1}\right)$ hold, then problem (1.1) possesses at least $k$ distinct pairs of weak solutions for $\lambda>\lambda_{k}, k=1,2, \ldots$, if the following assumptions are satisfied,

$\left(H_{2}\right)^{\prime}$. There are constants $c_{1}, c_{2}>0$ and $0<p<2$ such that

$$
F(t, x) \leq-\frac{\lambda}{2}|x|^{2}+c_{1}|x|^{p}+c_{2}, x \in R, t \in[0, T] .
$$


$\left(H_{4}\right)$. There exist $\varepsilon, r_{2}>0$, such that $F(t, x) \geq \varepsilon$, for $|x| \leq r_{2}$;

$\left(H_{5}\right) . F(t, x)=F(t,-x)$

Proof. Our aim is to apply Lemma 2.13 to the problem (1.1). So we verify all the assumptions of Lemma 2.13 hold.

From $F(t, 0)=0$ for all $t \in[0, T]$, it easy to see that $I(0)=0$. The assumption $\left(H_{5}\right)$ shows that $I(u)$ is even. From $\left(H_{2}\right)^{\prime}$, we have

$$
\begin{aligned}
I(u) & =\frac{1}{2} \int_{0}^{T}\left[\left.\left.a(t)\right|_{0} D_{t}^{\alpha} u(t)\right|^{2}-\lambda u^{2}(t)\right] d t-\int_{0}^{T} F(t, u(t)) d t \\
& \geq \frac{1}{2}\|u\|_{\alpha}^{2}-\frac{\lambda}{2}\|u\|_{L^{2}}^{2}+\frac{\lambda}{2}\|u\|_{L^{2}}^{2}-c_{1} T\|u\|_{\infty}^{p}-c_{2} T \\
& \geq \frac{1}{2}\|u\|_{\alpha}^{2}-c_{1} T\left(\frac{T^{\alpha-1 / 2}}{\Gamma(\alpha) \sqrt{a_{0}(2 \alpha-1)}}\|u\|_{\alpha}\right)^{p}-c_{2} T \\
& =\left[\frac{1}{2}-c_{1}\|u\|_{\alpha}^{p-2} T\left(\frac{T^{\alpha-1 / 2}}{\Gamma(\alpha) \sqrt{a_{0}(2 \alpha-1)}}\right)^{p}\right]\|u\|_{\alpha}^{2}-c_{2} T .
\end{aligned}
$$

Therefore $I(u)$ is bounded from below. Next, similarly to the proof of Theorem 3.2 , we can verify $I$ satisfies the (PS) condition.

From Theorem 3.1, we have the eigenfunctions $\left\{w_{j}\right\}$ of 3.1) corresponding to the eigenvalues $\left\{\lambda_{j}\right\}$. We select the space $E^{\prime}$ by

$$
E^{\prime}=\left\{u: u=\sum_{j=1}^{k} \alpha_{j} w_{j}, \alpha_{j} \in R \text { with } \sum_{j=1}^{k} \alpha_{j}^{2}=\frac{\Gamma(\alpha) \sqrt{a_{0}(2 \alpha-1)}}{T^{(\alpha-1) / 2}} r_{2}\right\} .
$$

Then the space $E^{\prime}$ is homeomorphic to the $k-1$ dimension unit sphere $S^{k-1}$ by an odd homeomorphic map which is injective, surjective and continuous with its inverse map is also continuous.

For all $u \in E^{\prime}$, from the definition of $u \in E^{\prime}$ and that $\left\{w_{j}\right\}$ are orthogonal basis, we can get

$$
\|u\|_{\alpha}=\frac{\Gamma(\alpha) \sqrt{a_{0}(2 \alpha-1)}}{T^{\alpha-1 / 2}} r_{2}
$$

together with $\left(H_{4}\right)$, it shows $\|u\|_{\infty} \leq r_{2}$. Also from the condition $\lambda>\lambda_{k}$, then one has

$$
\begin{aligned}
I(u) & =\frac{1}{2} \int_{0}^{T}\left[\left.\left.a(t)\right|_{0} D_{t}^{\alpha} u(t)\right|^{2}-\lambda u^{2}(t)\right] d t-\int_{0}^{T} F(t, u(t)) d t \\
& \leq \frac{1}{2}\|u\|_{\alpha}^{2}-\frac{\lambda}{2}\|u\|_{L^{2}}^{2}-\varepsilon T \\
& \leq \frac{1}{2}\|u\|_{\alpha}^{2}-\frac{\lambda k}{2}\|u\|_{L^{2}}^{2}-\varepsilon T .
\end{aligned}
$$

From Theorem 3.1, we have

$$
\lambda_{k} \geq \frac{\int_{0}^{T} a(t)\left|{ }_{0} D_{t}^{\alpha} u(t)\right|^{2} d t}{\int_{0}^{T} u^{2}(t) d t}=\frac{\|u\|_{\alpha}^{2}}{\|u\|_{L^{2}}^{2}}, \text { for }\|u\|_{\infty} \leq r_{2} .
$$

It should be noted that $r_{2}$ has no direct relation to $r_{1}$. Then it follows from (3.13) and (3.14) that $I(u)<0$, for $\|u\|_{\infty} \leq r_{2}$. Hence, it easy to get $\sup _{E}<0$. Then all the conditions of Lemma 2.13 are satisfied. From the assertion of Lemma 2.13, we know that $I(u)$ possesses at least $k$ distinct pairs of critical points which correspond to the weak solutions of the problem (1.1). 
Finally, we give some examples to illustrate the usefulness of our main result. Consider the following system of fractional differential equations.

\section{Example 3.4.}

$$
\left\{\begin{array}{l}
{ }_{t} D_{1}^{\frac{2}{3}}\left({ }_{0} D_{t}^{\frac{2}{3}} u(t)\right)=\lambda u+\nabla F(t, u(t)), \quad t \in[0,1], \\
u(0)=u(1)=0 .
\end{array}\right.
$$

Let $F(t, u(t))=-\frac{\lambda}{2}|u|^{2}+\left(c_{1}-1\right)|u|^{p}$, then $\left(H_{1}\right),\left(H_{2}\right)$ hold.

We choose $r_{1}<\frac{2\left(c_{1}-1\right)}{\lambda_{k}}$, it follows that $|u|^{2-p} \leq \frac{2\left(c_{1}-1\right)}{\lambda_{k}}$, which implies

$$
\frac{\lambda_{k}-\lambda}{2}|u|^{2} \leq-\frac{\lambda}{2}|u|^{2}+\left(c_{1}-1\right)|u|^{p}=F(t, u)
$$

Then $\left(H_{3}\right)$ is satisfied. According to Theorem 3.1. problem 3.15 has at least two non-trivial solution.

\section{Example 3.5.}

$$
\left\{\begin{array}{l}
{ }_{t} D_{1}^{\frac{3}{4}}\left({ }_{0} D_{t}^{\frac{3}{4}} u(t)\right)=2 u(t)+\nabla F(t, u(t)), \quad t \in[0,1], \\
u(0)=u(1)=0 .
\end{array}\right.
$$

Let $F(t, u(t))=-2 u^{2}+1$. Obviously $\left(H_{1}\right),\left(H_{2}\right)^{\prime}$, are satisfied with

$$
F(t, u(t))=-2 u^{2}+1 \leq-|u|^{2}+|u|+1.5
$$

where $\lambda=2, c_{1}=1, c_{2}=1.5, p=1$.

Let $\varepsilon=r_{2}=\frac{1}{2}>0$, then we have $F(t, u(t))=-2 u^{2}+1 \geq \frac{1}{2}$, for $|u| \leq \frac{1}{2}$. So $\left(H_{4}\right)$ holds. And $F(t, u(t))=-2 u^{2}+1$ is even, that is $\left(H_{5}\right)$ is satisfied. Then our results can be applied to problem (3.16), which shows problem $(3.16)$ has at least $k$ distinct pairs of distinct solutions.

\section{Acknowledgments}

The authors thank the referees for their careful reading of the manuscript and insightful comments, which help us to improve the quality of the paper. We would also like to acknowledge the valuable comments and suggestions from the editors, which vastly contribute to the perfection of the paper. This work is supported by National Natural Science Foundation of China (No.11001274, 11101126, 11261010, 11301153, 61070247), China Postdoctoral Science Foundation (No.20110491249), Key Program of Henan Province (132102210246), Key Technologies R \& D Program of Education Department of Henan Province (13B510001), Key Scientific and Technological Research Project of Department of Education of Henan Province (NO. 12B110006,14A510015), Youth Science Foundation of Henan University of Science and Technology(NO. 2012QN010), The Natural Science Foundation to cultivating innovation ability of Henan University of Science and Technology(NO. 2013ZCX020).

\section{References}

[1] B. Ahmad, J. J. Nieto, Existence results for a coupled system of nonlinear fractional differential equations with three-point boundary conditions, Comput. Math. Appl., 58 (2009), 1838-1843.1

[2] R. P. Agarwal, M. Benchohra, S. Hamani, A survey on existence results for boundary value problems of nonlinear fractional differential equations and inclusions, Acta Appl. Math., 109 (2010), 973-1033.1]

[3] C. Bai, Infinitely many solutions for a perturbed nonlinear fractional boundary value problem, Electron. J. Differential Equations, 2013 (2013), 12 pages.1, 1

[4] H. Brezis, L. Nirenberg, Remarks on finding critical points, Comm. Pure Appl. Math., 44 (1991), 939-963. 2.12

[5] J. Chen, X. H. Tang, Existence and multiplicity of solutions for some fractional boundary value problem via critical point theory, Abstr. Appl. Anal., 2012 (2012), 21 pages.1. 
[6] K. Diethelm, The Analysis of Fractional Differential Equation. An application-oriented exposition using differential operators of Caputo type, Lecture Notes in Mathematics, Springer-Verlag, Berlin, (2010). 1

[7] J. R. Graef, L. Kong, Positive solutions for a class of higher order boundary value problems with fractional q-derivatives, Appl. Math. Comput., 218 (2012), 9682-9689.1

[8] Z. Hu, W. Liu, J. Liu, Ground state solutions for a class of fractional differential equations with Dirichlet boundary value condition, Abstr. Appl. Anal., 2014 (2014), 7 pages.1]

[9] F. Jiao, Y. Zhou, Existence of solutions for a class of fractional boundary value problems via critical point theory, Comput. Math. Appl., 62 (2011), 1181-1199.1,2, 2.7, 2

[10] F. Jiao, Y. Zhou, Existence results for fractional boundary value problem via critical point theory, Internat. J. Bifur. Chaos Appl. Sci. Engrg., 22 (2012), 17 pages.1

[11] A. A. Kilbas, M. H. Srivastava, J. J. Trujillo, Theory and applications of fractional differential equations, Elsevier Science, Amesterdam, (2006). 1, 2.1, 2.2, 2.3, 2.4, 2.5, 2.6

[12] V. Lakshmikantham, S. Leela, J. V. Devi, Theory of fractional dynamic systems, Cambridge Scientific Publishers, Cambridge, (2009).1

[13] Y. N. Li, H. R. Sun, Q. G. Zhang, Existence of solutions to fractional boundary-value problems with a parameter, Electron. J. Differential Equations, 2013 (2013), 12 pages.1.

[14] Y. Liu, W. Zhang, X. Liu, A sufficient condition for the existence of a positive solution for a nonlinear fractional differential equation with the Riemann-Liouville derivative, Appl. Math. Lett., 25 (2012), 1986-1992.1.

[15] N. Nyamoradi, R. Rodríguez-López, On boundary Value Problems for Impulsive Fractional Differential equations, Appl. Math. Comput., 271 (2015), 874-892.1

[16] I. Podlubny, Fractional differential equations, Math. Sci. Eng., Academic Press, New York, (1999). 1

[17] P. H. Rabinowitz, Minimax methods in critical point theory with applications to differential equations, CBMS Regional Conf. Ser. in Math. American Mathematical Society, Providence, 65 (1986).2.13, 3

[18] R. Rodríguez-López, S. Tersian, Multiple solutions to boundary value problem for impulsive fractional differential equations, Fract. Calc. Appl. Anal., 17 (2014), 1016-1038.1

[19] H. R. Sun, Q. G. Zhang, Existence of solutions for a fractional boundary value problem via the Mountain Pass method and an iterative technique, Comput. Math. Appl., 64 (2012), 3436-3443.1.

[20] V. E. Tarasov, Fractional dynamics: application of fractional calculus to dynamics of particles, fields and media, Springer, Beijing, (2011).1

[21] C. Torres, Mountain pass solution for a fractional boundary value problem, J. Fract. Calc. Appl., 5 (2014), 1-10. 1

[22] J. R. Wang, Y. Zhou, A class of fractional evolution equations and optimal controls, Nonlinear Anal. Real World Appl., 12 (2011), 262-272.1

[23] Y. Zhao, H. Chen, B. Qin, Multiple solutions for a coupled system of nonlinear fractional differential equations via variational methods, Appl. Math. Comput., 257 (2015), 417-427.11

[24] Y. Zhou, F. Jiao, J. Li, Existence and uniqueness for fractional neutral differential equations with infinite delay, Nonlinear Anal., 7 (2009), 3249-3256.1 